\title{
INTERNAL QUALITY CONTROL OF AN ENZYME-LINKED IMMUNOASSAY FOR CRY1AB TOXIN DETECTION APPLIED IN ANIMAL TISSUES
}

\author{
E. TAKÁCS ${ }^{a}$ A. NAGY $Y^{b}$ É. GeLENCSÉR ${ }^{b}$ and A. SZÉKÁCS ${ }^{a *}$ \\ ${ }^{a}$ Agro-Environmental Research Institute and ${ }^{b}$ Food Science Research Institute, \\ National Agricultural Research and Innovation Centre, H-1022 Budapest, Herman Ottó út 15. Hungary
}

(Received: 21 July 2014; accepted: 8 September 2014)

\begin{abstract}
Reliable determination of microbial or transgenic Cry toxins is an essential issue in food and feed analyses, and enzyme-linked immunosorbent assays (ELISAs) are the method of choice for quantifying these toxins currently in food and environmental analysis. Internal Quality Control (IQC) is an indispensable method to assess accuracy, precision, and reproducibility of analytical measurements. To assess the utility of the ELISA method, IQC was performed on EnviroLogix Cry1Ab/Cry1Ac QualiPlate ELISA with manufacturer supplied analytical standards. Applicability of negative and positive controls (C- and $\mathrm{C}+$ ) was examined by Shewhart Control Charts for bias and Control Chart of the Range of Duplicates for precision. Linear regression (up to $5 \mathrm{ng} \mathrm{ml}^{-1}$ Cry1 $\mathrm{Ab}$ concentration) of the commercial ELISA kit was compared to sigmoid calibration (up to $60 \mathrm{ng} \mathrm{ml}^{-1} \mathrm{Cry} 1 \mathrm{Ab}$ concentration). For immunoassay optimization process, possible matrix effects in different liquid and solid vertebrate tissues were examined by determination of the limit of detection values in these matrices.
\end{abstract}

Keywords: internal quality control, ELISA, Cry1Ab, matrix effect, porcine tissue

Increasing application rates of various Bacillus thuringiensis (Bt) endotoxins in Bt-insecticides and in first generation genetically modified (GM) crops (Bt-plants) have emphasized the importance of an optimal analytical method for determination of Cry toxin dosages in studies on environmental risk assessment (ERA), resistance management, food and feed safety and quality control, and regulatory compliance. The most commonly applied Cry toxin is Cry1 Ab, specific to Lepidopteran insects. The protoxin form (131 kDa) of bacterial Cry1 Ab is produced by Bacillus thuringiensis var. kurstaki (Berliner) (HöFTE \& Whiteley, 1989; CERA, 2009). GM plants resistant to the European corn borer (Ostrinia nubilalis) produce a truncated (preactivated) form of Cry1 Ab toxin $(91 \mathrm{kDa})$, still larger in molecular size than the activated toxin (60-65 kDa) (De MaAgd et al., 1999; НilBeCK, 2001).

Due to instability of the target analyte and the laborious steps in chromatographic instrumental analysis, the analytical method of choice for quantitative detection of Cry toxins is enzyme-linked immunosorbent assay (ELISA) (WaLschus et al., 2002; Grothaus et al., 2006). Analytical difficulties and biological aspects of Cry toxin determination by ELISA systems include varying cross-reactivity of Cry toxin specific antibodies to various toxin forms, matrix effects in biological tissues, and uneven distribution of the toxin protein in the matrix (SzÉKÁCs et al., 2010; TAKÁcs et al., 2012). Commercial ELISA kits are designed for detection of $B t$ toxins in certain plant tissues (leaf and seed). However, ERA and food safety assessment require Cry toxin determination in other plant parts and in animal tissues as well.

* To whom correspondence should be addressed. Phone: +36-1-796-0401; e-mail: a.szekacs@cfri.hu 
Commercial ELISA kits include a protocol that determines the limit of detection (LOD). However, such LODs are appropriate only for matrices specified in the given protocols.

To study possible occurrence of Cry1Ab in porcine tissues, feeding studies with PR34N44 Bt-maize were performed (WALSH et al., 2011; BuZoianu et al., 2012; Walsh et al., 2012), and results indicated that appearance or detection success of $B t$ toxin in different tissues is not uniform. Cry1 Ab was positively detected in stomach content and digesta samples, but fell below the LOD for other tissues. Since, however, achievable signal intensities and LODs varied among these tissues, reliability of these analyses to eliminate false negative results is expedient to be assessed.

Internal quality control (IQC) is important in food analysis to verify that a laboratory produces reliable data consistently with minimum levels of error. Quality control is appropriate to detect both random and systematic errors that determine the precision and accuracy of the measurement, respectively. An excellent and widely used system of quality control is the application of Control Charts. In this study we report IQC of the data obtained in the above pig feeding studies using commercial EnviroLogix Cry1Ab/Cry1Ac QualiPlate ELISA. Control Chart of the Mean (Shewhart Chart) for the control of bias, and Control Chart of the Range of Duplicates (Range Chart) for the control of precision were performed to study precision and accuracy of the ELISA system. Reproducibility and accuracy of the linear calibration applied were examined and compared with sigmoid calibration that is the general calibration curve in sandwich ELISA systems. For immunoassay optimization process, possible matrix effects were examined in different liquid and solid porcine tissues by determining the LODs in these matrices.

\section{Materials and methods}

\subsection{ELISA method}

The commercial ELISA kit Cry1Ab/Cry1Ac QualiPlate (EnviroLogix Inc., Portland, ME, USA) was used to determine Cry1 Ab content according to manufacturer's protocol. Colour signals (optical density, OD) were read on a Thermo Multiskan Ascent 354 microplate reader (Thermo Electron Corp., Marietta, OH, USA).

\subsection{Standard curves}

For quantitative determination, analytical standards (supplied also by the manufacturer) were applied at $0,0.5,2.5$, and $5.0 \mathrm{ng} \mathrm{ml}^{-1}$ concentrations allowing linear calibration. Linear regression of EnviroLogix Cry1Ab/Cry1Ac QualiPlate in this concentration range was compared, using normalized OD values, to four parametric sigmoid calibration (concentration range up to $60 \mathrm{ng} \mathrm{ml}^{-1}$ ). Reliability of $\mathrm{C}+$ and $\mathrm{C}$ - were investigated by a statistical comparison of linear regression parameters in different methods: (1) linear regression without control points, (2) linear regression with C-, and (3) linear regression with C+.

\subsection{Shewhart Chart and Range Chart}

Shewhart Chart shows the relation with the normal distribution of the data around the mean. The Shewhart Chart consists of (1) points representing the statistic (mean) of measurements taken at different times, (2) a centre line at the value of the mean of statistic, (3) upper and 
lower warning limits (UWL, LWL): 2 standard deviations (SDs) above and below the centre line and (4) upper and lower control limits (UCL, LCL): 3 SDs from the centre line.

Range Chart shows (1) the difference between replicates of each sample analysis, as well as the warning limit (WL) and (3) the control limit (CL), drawn at 2 and 3 SDs from the mean of differences, respectively. The graph is single-sided as the lowest observable value of the difference is zero.

Both chart analyses are under statistical control if 95\% of results are within WLs and 99.7\% of the results are within CLs. Any observations outside the limits, or systematic patterns within, suggest the introduction of a new source of variation (SHEWHART, 1931; FAO, 1998).

To assess analytical reliability, accuracy and precision of the ELISA system, Shewart Charts and Range Charts were plotted to each of the analytical standards, as well as negative $(\mathrm{C}-)$ and positive $(\mathrm{C}+)$ controls. $\mathrm{C}+$ and $\mathrm{C}-$ were determined by individual measurements and by linear regression in 36 individual measurements.

\subsection{Matrix effect}

Results of Cry1Ab toxin concentrations determined in porcine tissue samples were available from pig feeding studies (Walsh et al., 2011, 2012; Buzoianu et al., 2012). Organ samples were heart, kidney, spleen, liver, muscle, lymph node, brain, and serum of pigs fed with GM MON 810 (PR34N44) and non-GM isogenic maize (PR34N43). Digesta samples were collected from stomach, cecum and colon. For characterization of matrix effects in different tissues matrix-to-matrix LODs were determined. Cry1Ab determination was performed on 10-10 animal samples. Relative absorbance $\left(\mathrm{OD}_{\text {rel }}\right)$ values were calculated for each tissue by comparing them to the highest standard calibrator solution (5 $\left.\mathrm{ng} \mathrm{ml}^{-1}\right)\left(\mathrm{OD} / \mathrm{OD}_{\max }\right)$.

\subsection{Statistical analysis}

Distributions of data were evaluated before analysis using normal probability plot and Shapiro-Wilk normality test (SHAPIRo \& WiLK, 1965). Differences among different linear regressions were analysed by Student's $t$-test or Mann-Whitney U-test at probability level of 0.01 . Equality of variance, as requirement in application of $t$-test, among sample groups was also monitored.

\section{Results and discussion}

\subsection{Standard curves}

The EnviroLogix Cry1Ab/Cry1Ac QuantiPlate ELISA kit was unexpectedly withdrawn from the market in 2005, and was replaced with a qualitative EnviroLogix Cry1Ab/Cry1Ac QualiPlate. The latter system provides only C+ control solution, but not analytical standards, yet is feasible for quantitative determination with manufacturer supplied standards in the concentration range of 0.5 to $5 \mathrm{ng} \mathrm{ml}^{-1}$ and $\mathrm{C}-$ control solution. Blank was used for $0.0 \mathrm{ng} \mathrm{ml}^{-1}$ concentration standard. Parameters of the average normalized linear calibration, determined in 36 individual measurements, were: (i) slope: $19.839 \pm 0.197$, (ii) y-intercept: $0.644 \pm 1.363$, and (iii) $\mathrm{R}^{2}$ : $0.9997 \pm 0.0034$ (Fig. 1. insert). It is concluded that both $\mathrm{C}+$ and $\mathrm{C}$ - do not modify the regression parameters significantly, thus both control points are appropriate to provide reliable measurement. ELISA systems are typically calibrated with four-parameter logistic fit, providing a sigmoid standard curve (RODBARD, 1981). To achieve lower LODs, the 
commercial ELISA systems utilize linear regression to the standard curve on the concentration range of the lower plateau of the sigmoid curve. By such shifting of the LOD downwards, accuracy of the measurement is deteriorating. The most reliable part of the sigmoid calibration curve is the middle, quasi-linear section, thus accuracy and reliability of determination is the best at the inflexion point $\left(\mathrm{IC}_{50}\right)$. Concentration range of 0 to $5 \mathrm{ng} \mathrm{ml}^{-1}$ and 0 to $60 \mathrm{ng} \mathrm{ml}^{-1}$ for linear and sigmoid calibration, respectively, are compared in Figure 1.

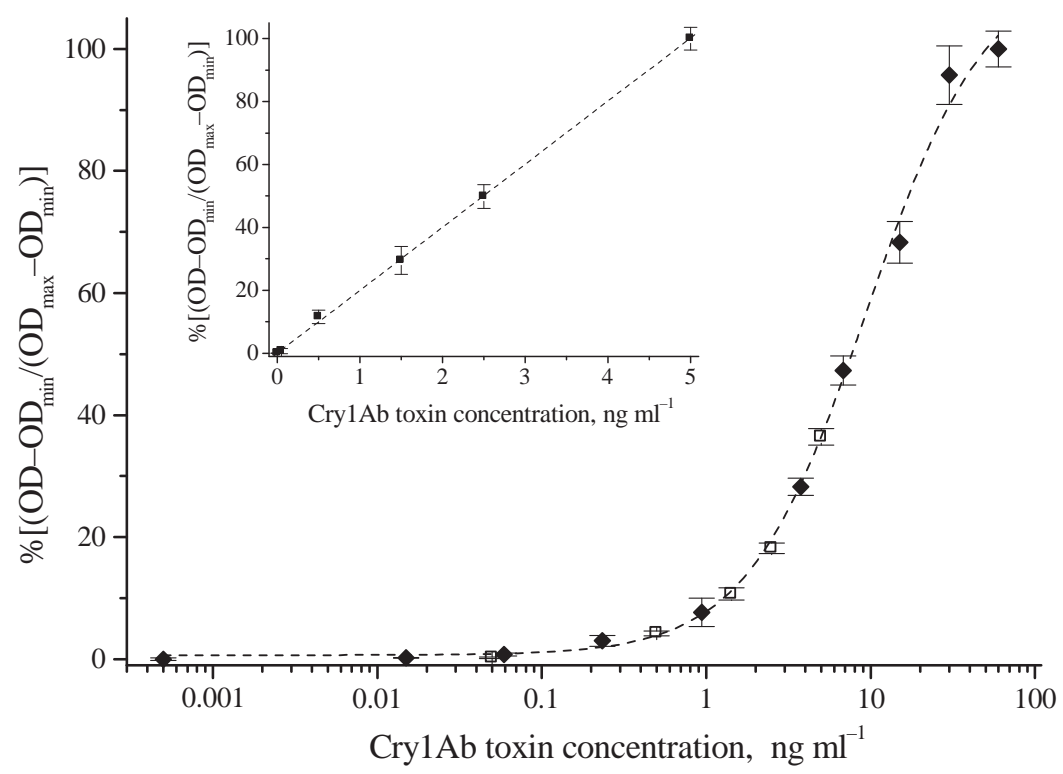

Fig. 1. Normalized average sigmoid and linear (insert) regression of 36 individual measurement by the EnviroLogix Cry1Ab/Cry1Ac QualiPlate kit. Data points used for linear regression ( $\square$ ) show excellent fit on the sigmoid curve (

\subsection{Shewhart Chart and Range Chart}

Shewhart Charts and Range Charts (for the control of bias and precision, respectively) were plotted to each analytical standards $\left(0,0.5,2.5\right.$, and $\left.5.0 \mathrm{ng} \mathrm{ml}^{-1}\right)$ and control samples $(\mathrm{C}+$ and C-) of the Cry1Ab/Cry1Ac QualiPlate ELISA. These control charts for C- and C+ as control points are depicted in Figure 2. For C-, 3 points from 36 individual measurements were found to fall outside of UWL or LWL, of which 1 point fell outside of UCL as well in the Shewhart Chart (Fig. 2A). In consequence, only $91.7 \%$ of the results were between the upper and lower warning limits, meaning that the analysis is out of control. In case of Range Chart, two outlier points were determined, one outside the WL and one outside the CL (Fig. 2B). Both points found outside of the control limits in these two charts were the result of the same measurement procedure. Corresponding mean, UCL, UWL, LWL, and LCL values of the Shewhart Charts are detailed in Table 1 and results of the Range Chart in Table 2. 

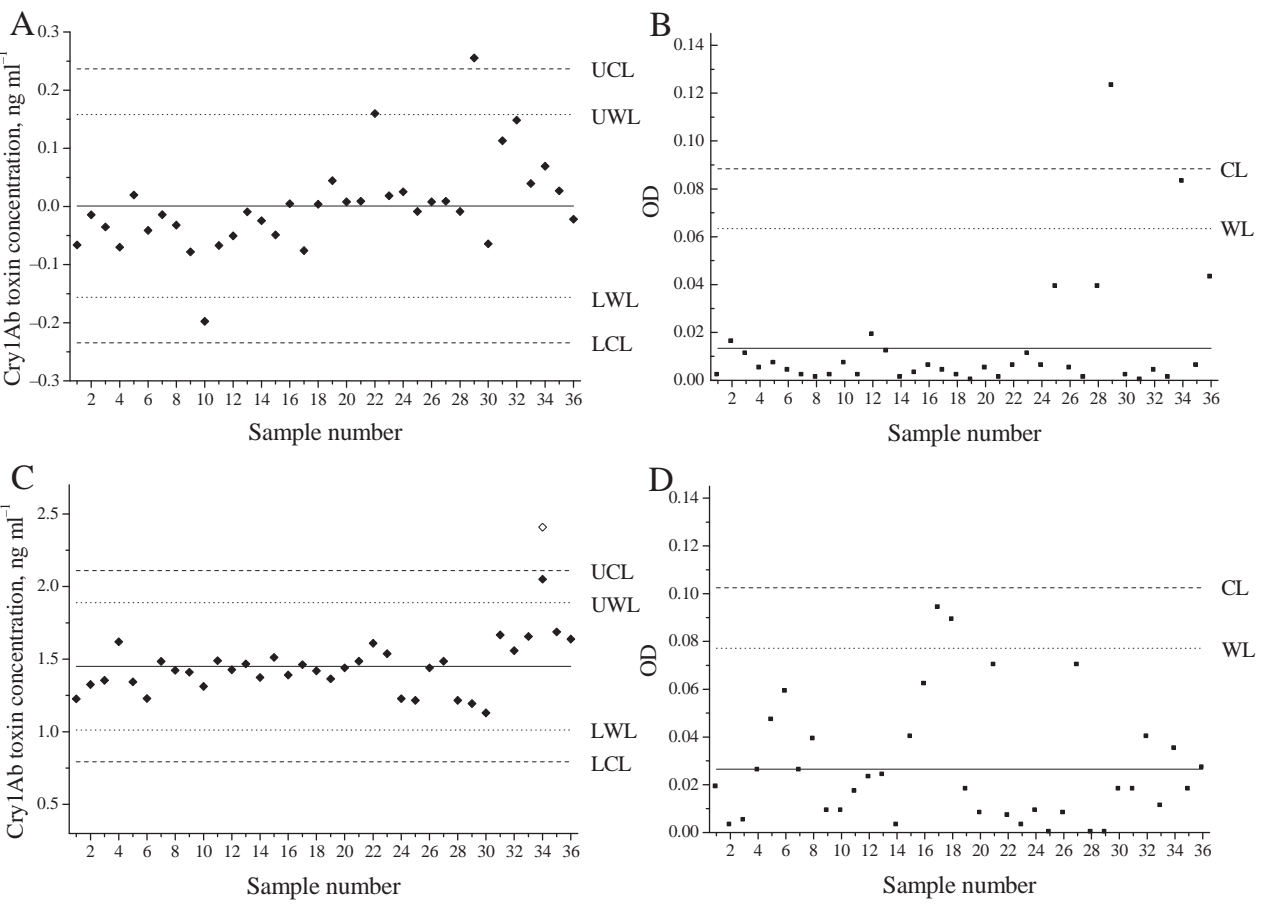

Fig. 2. Internal Quality Control of Cry1Ab determination by the EnviroLogix Cry1Ab/Cry1Ac QualiPlate kit using the negative control (C-) sample (A, B) and the positive control $(\mathrm{C}+$ ) sample $(\mathrm{C}, \mathrm{D})$ as reference value. Shewhart Charts $(A, C)$ indicate bias of given reference values from the average, Range Charts $(B, D)$ show differences between duplicates within each determination (ELISA plate)

Table 1. Detailed values of statistical control limits of Shewhart Charts, i.e. upper control limit (UCL), upper warning limit (UWL), lower warning limit (LWL), and lower control limit (LCL) using negative control (C-) and positive control $(\mathrm{C}+)$ samples as control point

\begin{tabular}{cccccc}
\hline & \multicolumn{5}{c}{ Cry1Ab toxin concentrations, ng ml $^{-1}$} \\
\cline { 2 - 5 } & Average & UCL & UWL & LWL & LCL \\
\hline C- & $0.001 \pm 0.079$ & 0.237 & 0.158 & -0.156 & -0.235 \\
C + & $1.451 \pm 0.219$ & 2.109 & 1.890 & 1.012 & 0.793 \\
\hline
\end{tabular}

Table 2. Range Chart. Average difference ODs of duplicates among 36 individual measurements, control limits $(\mathrm{CL})$, and warning limits (WL) using negative control $(\mathrm{C}-$ ) and positive control $(\mathrm{C}+)$ samples as control point

\begin{tabular}{cccc}
\hline & \multicolumn{3}{c}{ Optical density (OD) } \\
\cline { 2 - 4 } & Average & CL & WL \\
\hline $\mathrm{C}-$ & 0.013 & 0.088 & 0.063 \\
$\mathrm{C}+$ & 0.027 & 0.103 & 0.077 \\
\hline
\end{tabular}

It is known that nominal Cry1 Ab concentration of $\mathrm{C}-$ is $0 \mathrm{ng} \mathrm{ml}^{-1}$. The average calculated concentration of the negative control was $0.001 \mathrm{ng} \mathrm{ml}^{-1}$. Although difference from the nominal value occurred at the fourth decimal place, its relative SD was extremely high 
8543.5\%. This vast error, however, is due to the low mean value, and not the actual SD. Among individual measurements relative SDs ranged from $0.00 \%$ to $141.42 \%$ for OD values, and from $-1326.95 \%$ to $595.35 \%$ for calculated concentrations by given linear regression. In consequence $\mathrm{C}$ - is evaluated as inappropriate to be used as reference dilution, both by its IQC and high relative SD.

For C+ only one point was outside of the UCL in the Shewhart Chart, $97.2 \%$ of the results were located between the UCL and LCL (Fig. 2C). Only one outlier point was found not to be systematic error in analysis, but human mistake (e.g. pipetting error). These results clearly indicate that the Shewhart Chart is feasible not only for determining systematic errors in analytical measurements, but also for detecting and eliminating individual or human errors. After elimination or correction of the single outlier point, all remaining points fell between the thus modified warning limits. The calculated average Cry1Ab concentration was 1.45 with $15.11 \%$ relative SD. In case of Range Chart, all point feel below the CL with 2 points outside the WL (Fig. 2D). Relative errors in individual measurements ranged from $0.00 \%$ to $14.05 \%$ for OD values and from $0.00 \%$ to $28.80 \%$ for calculated concentrations.

\subsection{Matrix effects}

ELISAs are often used to detect antigens in heterogeneous samples like plant or animal tissues. Examination of matrix effects in porcine plasma samples resulted lower LOD values than those detected at $0 \mathrm{ng} \mathrm{ml}^{-1}$ standard concentration. In the development of an immunoassay the first step is the coating process, where specific antibodies or antigens are fixed on the surface of microplate wells, and the second step is to effectively block the remaining binding sites to prevent non-specific binding of different matrix components. Blocking is not always complete, thus, slight background signals can be detected for blank samples due to limited binding of other molecules (e.g. enzyme conjugate, non-target proteins) to empty places in the microplate wells. Plasma samples contain various antibodies being present in the vertebrate blood system, and these antibodies may particularly occupy empty surfaces of the wells.

Average relative $\mathrm{OD}\left(\mathrm{OD}_{\mathrm{rel}}\right)$ and average $\mathrm{LOD}$ values of three independent studies are presented in Fig. 3. The highest LOD values, $7.68 \pm 6.54 \mathrm{ng} \mathrm{ml}^{-1}$, were determined in muscle samples. The unexpectedly high SD means that this tissue proved to be difficult matrix in Cry1Ab detection. This difficulty may cause an important problem in food safety quality control studies, where controlling of the GMO-free status is necessary. Relatively high LOD values were determined also in digesta samples, and SDs among different studies were high as well. This signal, however, corresponds to detection of Cry1 Ab toxin in the plant sample digested to various degrees in the pig intestine, and high variability may occur due to different decomposition rates in digesta samples. 
relOD 口

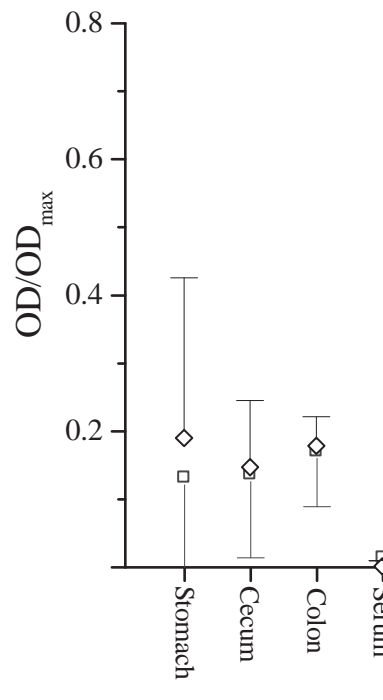

$\diamond \quad$ LOD

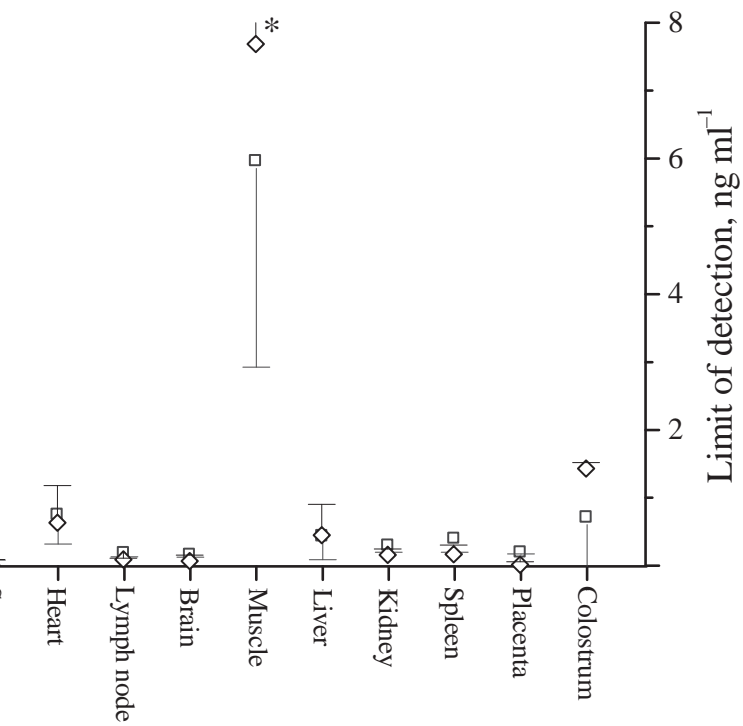

Porcine tissue/organ

Fig. 3. Limit of detection (LOD) and relative OD ( $\left.\mathrm{OD}_{\text {rel }}\right)$ values for different porcine tissues (stomach, cecum, colon, serum, heart, lymph node, brain, muscle, liver, kidney, spleen, placenta, and colostrum) determined by the EnviroLogix Cry1Ab/Cry1Ac QualiPlate kit. (* The full error bar of the LOD for muscle tissue, $\pm 6,542 \mathrm{ng} \mathrm{ml}^{-1}$, is not shown due to graph scaling.) relOD: $\square$; LOD: $\diamond$

\section{Conclusions}

In this study analytical accuracy, reliability, and reproducibility of the EnviroLogix Cry1Ab/ Cry1Ac QualiPlate ELISA kit were examined by IQC. This ELISA system was proven to be appropriate for quantitative determination with manufacturer supplied standards. Although linear regression in analytical standard calibration provides better LODs due to its lower concentration range ( 0 to $5 \mathrm{ng} \mathrm{ml}^{-1}$ ), analytical reliability and accuracy of determination are poorer than in sigmoid calibration. According to our IQC results, manufacturer supplied C+ is appropriate for food safety quality control, but C-is not reliable as control point at $0 \mathrm{ng} \mathrm{ml}^{-1}$ Cry1 Ab concentration. The Shewhart Chart was also proven to be effective in the identification of human errors in the measurement process. Matrix effects among porcine tissues are different, high LOD values were determined for muscle tissue, that may be important and a difficulty in food safety control.

This study was supported by FP7 project \#211820 GMSAFood “GM post market monitoring” and by project AD005 "Development and application of analytical methods for the detection of Cry toxin proteins in plant and animal tissues” by the Hungarian Ministry of Agriculture. 


\section{References}

Buzoianu, S.B., Walsh, M.C., Ujhelyi, G., Szabó, E., Rea, M.C., O’Donovan, O., Nagy, A., Ross, R.P., Gardiner, G.E., Gelencsér, É. \& LAwlor, P.G. (2012): Effects of feeding Bt maize to sows during gestation and lactation on maternal and offspring immunity and fate of transgenic material. PLoS ONE, 7, e47851. doi:10.1371/ journal.pone.0047851.

CERA (2009): GM Crop Database. MON-ØØ81Ø-6 (MON810). CERA, Center For Environmental Risk Assessment Washington D.C., USA, Available at http://www.cera-gmc.org/?action=gm_crop_database\&mode= Submit\&evidx=9 (last accessed: 1 September 2014)

De Maagd, R.A., Bosch, D. \& Stiekema, W. (1999): Bacillus thuringiensis toxin mediated insect resistance in plants. Trends Plant Sci., 4, 9-13.

FAO (1998): Guidelines for quality management in soil and plant laboratories. Food and Agricultural Organization, FAO Soils Bulletin, 74.

Grothaus, G.D., Bandla, M., Currier, T., Giroux, R., Jenkins, G.R., Lipp, M., Shan, G., Stave, J.W. \& Pantella, V. (2006): Immunoassay as an analytical tool in agricultural biotechnology. J. AOAC Int. 89, 913-928.

Hirbeck, A. (2001): Implications of transgenic, insecticidal plants for insect and plant biodiversity. Perspect. Plant Ecol. Evol. Syst., 4, 43-61.

HöFTe, H. \& Whiteley, H. (1989): Insecticidal crystal proteins of Bacillus thuringiensis. Microbiol. Rev., 53, $242-$ 255.

RodBard, D. (1981): Mathematics and statistics of ligand assays: an illustrated guide. -in: LANGAN, J. \& CLAPP, J.J. (Eds) Ligand assay: Analysis of international developments on isotopic and nonisotopic immunoassay. Masson, New York, USA, pp. 45-101.

Shapiro, S.S. \& WiLK, M.B. (1965): An analysis of variance test for normality (complete samples). Biometrika, 52, 591-611.

SHEWHART, W.A. (1931): Economic control of quality manufactured product. D. Van Nostrand Company, Inc., New York, Republished in 1980 by the American Society of Quality Control, 501 pages.

Székács, A., Lauber, É., Takács, E. \& Darvas, B. (2010): Detection of Cry1Ab toxin in the leaves of MON810 transgenic maize. Anal. Bioanal. Chem. 396, 2203-2211.

TAKÁCs, E., DARVAS, B. \& SzÉKÁCS, A. (2012): Analytical difficulties and certain biological aspects of Cry1Ab toxin determination in MON 810 genetically modified maize. Acta Phytopathol. Entomol. Hung., 47, 293-306.

Walsh, M.C., Buzoianu, S.G., Gardiner, G.E., Rea, M.C., Gelencsér, É., JÁnosi, A., Epstein, M.M., Ross, R.P. \& LAwLOR, P.G. (2011): Fate of transgenic DNA from orally administered Bt MON810 maize and effects on immune response and growth in pigs. PLoS ONE, 6, e27177. doi:10.1371/journal.pone.0027177

Walsh, M.C., Buzoianu, S.G., Rea, M.C., O’Donovan, O., Gelencsér, É, Újhelyi, G., Ross, R.P., Gardiner, G.E. \& LAWLOR, P.G. (2012): Effects of feeding Bt MON810 maize to pigs for 110 days on peripheral immune response and digestive fate of the cry1Ab gene and truncated Bt toxin. PLoS ONE, 7: e36141. doi:10.1371/ journal.pone.0036141

Walschus, U., Witt, S. \& Wittmann, C. (2002): Development of monoclonal antibodies against Cry1Ab protein from Bacillus thuringiensis and their application in an ELISA for detection of transgenic Bt-maize. Food Agric. Immunol., 14, 231-240. 